\title{
In Silico Molecular Docking of Lavandula Angustifolia Mill's Compounds Along with a Number of Antianxiety Drugs with GABAA Receptor for Reduce Stress
}

\author{
ALI KAZEMI BABAHEYDARII* and EHSAN HEIDARI SOURESHJANI ${ }^{2}$ \\ ${ }^{1}$ Faculty of Science, Department of Chemistry, Islamic Azad University, \\ Shahrekord Branch, Shahrekord, Iran. \\ ${ }^{2}$ Department of Biological and Young Researchers and Elites Club,Islamic Azad University, \\ Shahrekord Branch, Shahrekord, Iran. \\ ${ }^{*}$ Corresponding author E-mail: kazemiali@iaushk.ac.ir \\ http://dx.doi.org/10.13005/ojc/300270
}

(Received: February 15, 2014; Accepted: March 08, 2014)

\begin{abstract}
GABAA receptor is hetero-oligomeric $\mathrm{Cl}$ - channel that is elective blocked by the alkaloid bicuculline and modulated by steroids, barbiturates and benzodiazepines. The anticonvulsant activity of Diazepam, Amobarbital and Phenobarbital may be mediated in Section by enhancement of inhibition involving y-aminobutyric acid (GABA). Lavender is one of the maximum effective medicinal plants various therapeutic effects of lavender, so as sedative, spasmolytic, antiviral, and antibacterial activities have been reportage. The molecular docking analyses done indicate the highly and effectively interactions between GABA and the Lavandula angustifolia Mill compounds. Ligand Lavandula angustifolia Mill compounds with GABAA are safer and milder with fewer or no side effects than the drugs currently used in the remedy of lessening high Stress which can be better for the development of new therapeutics to blocked GABAA lessening stress. Results conûrm all the Lavandula angustifolia Mill compounds were good binding energy when compared with the binging energies of Diazepam, Amobarbital and Phenobarbital.
\end{abstract}

Key words: Amobarbital, Docking, Diazepam, Lavandula angustifolia Mill, Phenobarbital, GABAA receptor.

\section{INTRODUCTION}

\section{Background}

Automated molecular docking methods have frequently been used to predict energetically favorable conformations and orientations of ligands in the structure of proteins. These methods combine search algorithms to generate different dockings and scoring functions to determine the tightness of protein-ligand interactions ${ }^{1}$.

True lavender (Lavandula angustifolia Mill. syn. L. officinalis Chaix) is a perennial shrub of the family Lamiaceae. It is native to southern Europe 
and the Mediterranean area and is commercially cultivated in France, Spain, Portugal Hungary, the UK, Bulgaria, Australia, China and the USA ${ }^{2}$. This plant is cultivated primarily for its aromatic inflorescence from which the essential oil is isolated, although its fresh and dried flowers are also marketed ${ }^{3}$. Lavender oil is known for its excellent aroma and is extensively used in the perfumery, flavor and cosmetic industries. The oil is known to possess sedative, carminative, anti-depressive and anti-inflammatory properties. It was also found to be active against many species of bacteria, including those resistant to antibiotics, such as methicillin-resistant Staphylococcus aureus and vancomycin-resistant Enterococcus ${ }^{4}$. Lavender oil is known for to be an effective antifungal agent against Aspergillus nidulans and Trichophyton Mentagrophytes ${ }^{5}$. Lavender's essential oil is commonly used in aromatherapy and massage. Its major clinical benefits are on the central nervous system. Many studies conducted on both animals and humans support issues as a sedative, anxiolytic and mood modulator. Particular chemical constituents of lavender have potent anticarcinogenic and analgesic properties. Aromatherapy with lavender oil has been recommended to treat a wide range of ailments including stress, anxiety depression, fatigue, motion sickness, and hypertension ${ }^{6}$.

In the mammalian focal nervous system, GABA is the main inhibitory neurotransmitter. Conformational restricted analogues of GABA have been used to assistance identify three basic GABA receptors which are called GABAA, GABAB and $G A B C$ receptors. Lavender's essential oil is that exerts anxiolytic, sedative, anticonvulsant and most of these effects are thought to result from a facilitation of the action of gamma aminobutyric acid (GABA), an inhibitory neurotransmitter in the central nervous system. $\gamma$-Aminobutyrate (GABA) is a major inhibitory neurotransmitter in the adult mammalian brain. GABA is also considered to be a multifunctional molecule that has different situational functions in the central nervoussystem, the peripheral nervous system, and in some nonneuronal tissues. GABA is formed within GABAergic axon terminals and released into the synapse, where it acts at one of two types of receptor GABAA which controls chloride entry into the cell, and $G A B A B$, which increases potassium conductance, decreases calcium entry, and inhibits the presynaptic release of other neurotransmitters 7. In the brain, $17-20 \%$ of all neurons are GABAergic and most of the physiological activities of GABA are generated through GABAA receptors (GABAA-Rs). These ionotropic receptors or ligand-gated ion channel (LGIC) are chloride anion (Cl-) channels that can be opened and activated by the endogenous neurotransmitter GABA and several drug classes, including benzodiazepines, barbiturates, steroids, anesthetics and convulsants. As the primary receptor for GABA in the CNS, GABAA receptors are involved in a variety of behavioral and cognitive processes ${ }^{8}$.

Diazepam, Amobarbital and Phenobarbital is commonly drug used to treat and reduce anxiety, panic attacks, and insomnia. Diazepam first was synthesized by Leo Sternbach ${ }^{9}$ to treat a wide range of conditions, and has been one of the most frequently prescribed medications in the World since its launch in 1963. The pharmacological action of Diazepam, Amobarbital and Phenobarbital increases the effect of the neurotransmitter GABA by binding to the benzodiazepine site on the GABAA receptor via the constituent chlorine atom ${ }^{10}$. The side effects of Diazepam, Amobarbital and Phenobarbital include sedation, excitement, rage, or seizure in epileptics which are calmed may persist for at least six months. These synthetic drugs also have physical dependence potential and can cause serious problems of physical dependence with long term use. Because of the role of Diazepam, Amobarbital and Phenobarbital as a positive allosteric modulator of GABA when it binds to benzodiazepine receptors it causes inhibitory effects. This arises from the hyperpolarization of the post-synaptic membrane owing to the control exerted over negative chloride ions by GABAA receptors. The purpose of this experiment was to evaluate the activity and reduce nerve pain sedation by lavender plants and its effects on the central nervous system of the GABAA receptor.

\section{MATERIAL AND METHODS}

The protein cholesteryl esterase was retrieved from the RCSB protein databank 
(www.rcsb.org/pdb) and saved in pdb file format (Fig.1). The active site SER194 identified through Pocker-Finder and Q-Site Finder.

\section{Molecular docking}

Docking is the process by which two molecules fit together in three-dimensional space and molecular docking is a useful tool in structural molecular biology and computer-assisted drug design and bioinformatics is seen as an emerging field with the potential to significantly improve how drugs are found and brought to the clinical trials and eventually released to the marketplace. This method is widely used to predict the predominant binding mode(s) of a ligand with a protein. Besides, reliability in docking of ligand molecules to protein or other targets is an important challenge for molecular modeling. One applications of the docking technique includes the prediction of the binding mode of novel drugs. Docking permits the scientist to virtually screen a database of compounds and bode the strongest binders based on variant scoring functions. The docking analyses were carried by HEX and AutoDock docking software.

Hex calculates protein-ligand docking, and it can superpose pairs of molecules using only witting of their 3D shapes. It avails Spherical Polar Fourier (SPF) correlations to accelerate the calculations and its one of the few docking programs which has built in graphics to view the effect ${ }^{11}$. It explores ways in which two molecules, such as Lavandula angustifolia Mill compounds and GABAA receptor (Fig.1) fit together and dock to each other well. The collection of Lavandula angustifolia Mill compounds and GABAA receptor was discovered via docking and their relative stabilities were evaluated using molecular dynamics and their binding affinities, using free energy simulations. The parameters applied for the docking process via HEX docking were:

$\begin{array}{ll}\text { - } & \text { Correlation type }- \text { Shape only } \\ \text { - } & \text { GFT Mode }-3 D \text { fast life } \\ \text { - } & \text { Receptor range }-180 \\ \text { - } & \text { Ligand Range }-180 \\ \text { - } & \text { Twist range }-360 \\ \text { - } & \text { Distance Range }-40\end{array}$

AutoDock4.2, a similar suite of programs involved genetic algorithm, was employed to gain an insight into the lavender's compounds bindings with GABAA receptor ${ }^{12-14}$. This program is designed to predict how small molecules, such as drug candidates, bind to a receptor. For the ligand conformational searches the Lamarckian genetic algorithm (LGA) was selected. 3D atomic coordinates of GABAA receptor were obtained from the Protein Data bank ${ }^{15}$ and prepared for docking. All nonpolar hydrogens were merged and partial atomic charges were assigned using the GasteigerMarsili method. Different grid boxes with different grid points in size with a grid-point spacing of 0.375 $\AA$ were considered for docking. Each map was centered such that it covered the entire protein including all possible binding sites. The 3D structures of ligands were at first built using ArgusLab4.0.1 molecular builder and then optimized using the GAUSSIAN package using B3LYP with $6-31 \mathrm{~g}^{*}$ basis set ${ }^{16,17}$. All rotatable bonds were assigned for the ligands, and also partial charges and nonpolar hydrogens were calculated and merged using the above mentioned method for the receptor.

\section{RESULTS AND DISCUSSION}

\section{Hex Results}

Molecular docking results of the Lavandula angustifolia Mill compounds ligand (Linalool, linalyl acetate, lavandulyl acetate, $\alpha$ terpineol and geranyl acetate) with the GABAA nerve receptors obtained using Hex are shown in Table 1.

Prediction of interaction energies between ligand and receptor has been a major challenge for molecular docking. Hex uses scoring algorithms to calculate these energy values of the docked complexes and stability of the docked complexes increases with decrease in energy value. While comparing the results obtained with all the Lavandula angustifolia Mill compounds ligand it is prominent that all of them show better stability when docked with GABAA nerve receptor. It is noted that the energy value is comparatively less for the modeled structure of geranyl acetate than other Lavandula angustifolia Mill compounds with the energy maching value of $(-256.12)$ hence conforming highest stability (Fig 2). 
Auto dock results

To predict the appropriate interaction of Diazepam, Amobarbital and Lavender compounds with GABAA receptor, the ligand molecules were docked with the target protein using Autodock. The analysis command in the docking parameter file in

Table 1: Results of molecular docking of the the Lavandula angustifolia Mill compounds with GABAA nerve receptors using the hex along with intermolecular bonding information

\begin{tabular}{llcccc}
\hline Receptor & Ligand & R Docking & E Docking & R Maching & E Maching \\
\hline Diazpeam & 11.6 & -295.80 & 6.4 & -391.56 \\
Amobarbital & 12 & -167.00 & 9.2 & -285.27 \\
Phenobarbital & 12.98 & -161.45 & 8.7 & -273.13 \\
& Linalool & 10.4 & -209.85 & 7.2 & -359.42 \\
linalylacetate & 10.4 & -248.29 & 8.2 & -361.88 \\
lavandulyl acetate & 10.8 & -228.21 & 7.2 & -372.72 \\
a-terpineol & 11.2 & -188.16 & 6.4 & -347.48 \\
Geranyl acetate & 9.6 & -256.12 & 6.4 & -397.31 \\
\hline
\end{tabular}

Table 2: Result of energy minimization after 10 runs of docking

\begin{tabular}{lccccccc}
\hline Compound & $\begin{array}{c}\text { No. of } \\
\text { atoms }\end{array}$ & $\begin{array}{c}\text { Eb } \\
(\mathbf{k c a l} / \mathbf{m o l})\end{array}$ & $\begin{array}{c}\text { kl } \\
(\mathbf{u M})\end{array}$ & $\begin{array}{c}\text { Intermolecular } \\
\text { Energy }\end{array}$ & $\begin{array}{c}\text { Internal } \\
\text { energy }\end{array}$ & $\begin{array}{c}\text { Torsional } \\
\text { energy }\end{array}$ & $\begin{array}{c}\text { Unbounded } \\
\text { External } \\
\text { energy }\end{array}$ \\
\hline$\alpha$-terpineol & 12 & -5.99 & 40.59 & -6.59 & -0.12 & 0.6 & -0.12 \\
Diazepam & 28 & -6.5 & 17.09 & -6.8 & -0.22 & 0.3 & -0.22 \\
Geranyl acetate & 14 & -6.29 & 24.46 & -8.08 & -0.34 & 1.79 & -0.34 \\
Lavandulyl acetate & 14 & -6.03 & 37.92 & -7.82 & -0.52 & 1.79 & -0.52 \\
Linalool & 12 & -5.72 & 64.58 & -7.21 & -0.17 & 1.49 & -0.17 \\
Linalyl acetate & 14 & -6.1 & 33.54 & -7.89 & -0.49 & 1.79 & -0.49 \\
Amobarbital & 21 & -3.22 & 16.00 & -4.89 & -0.17 & 2.09 & -0.17 \\
Phenobarbital & 22 & -4.65 & 39.6 & -6.14 & -0.08 & 1.49 & 0.08 \\
\hline
\end{tabular}

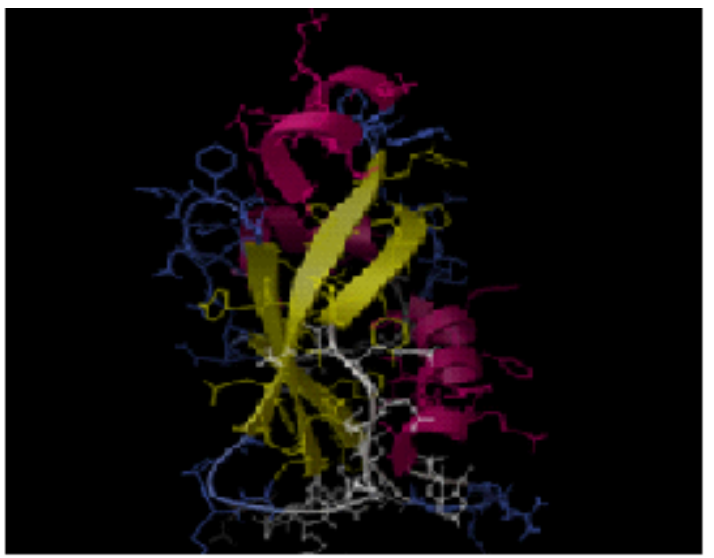

Fig. 1: GABAA receptor

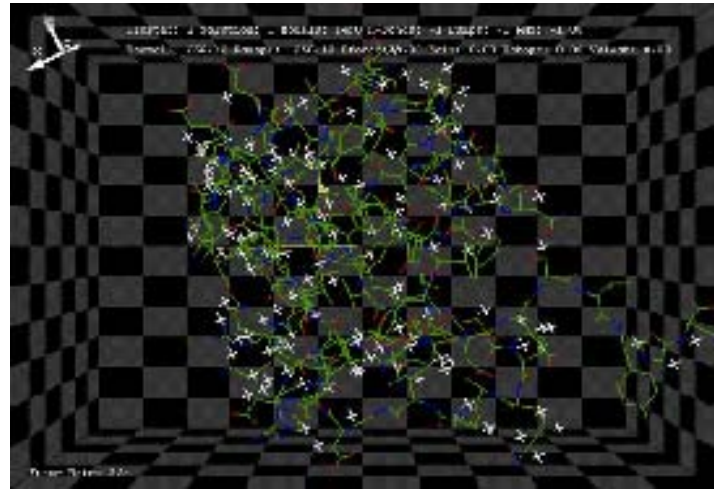

Fig. 2: Interaction and binding energy of geranyl acetate with $\mathrm{GABA}_{\mathrm{A}}$ nerve receptor 
this software causes AutoDock to perform a cluster analysis of the different docked conformations and find the minimum energy in each run. Every docking contains some useful knowledge which includes Binding Energy (Eb) which is the sum of the Intermolecular Energy, the Torsional Energy, and the Internal Energy which are reported in table 2. Since every ligand has different number of atoms, it is also mentioned in this table as No. of atoms. kl is the dissociation constant for a ligand with this Binding Energy, Cluster RMS is the root mean square difference in coordinates between this conformation and the cluster reference. In all of our cases it was found zero, because the selected conformations are the cluster reference. Reference RMS is the rms difference between this structure and the input structure. The study and compare of all dockings indicates that Diazepam and Geranyl acetate's ligands with the receptor protein, GABAA, gave the best docking results. According to table 2, these two complexes have the lowest amount of binding energy which is one important criterion. In

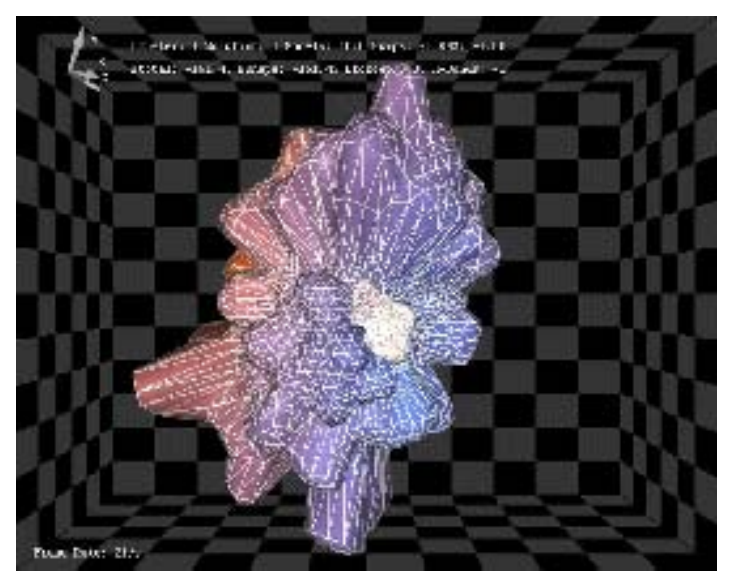

Fig. 3: Harmonic surfaces Interaction and binding energy of Phenobarbital with $\mathrm{GABA}_{\mathrm{A}}$ nerve receptor figure 4, the best docked position of Geranyl acetate on the GABAA receptor is shown.

\section{CONCLUSION}

The results emerged from the molecular docking of GABAA provide partial evidence for the traditional medicinal property for reduce stress. In the present study, we considered six different GABAA-ligand complexes using Hex and AutoDock software. The study provides the computational result of interaction between Lavender plants compounds concomitant Diazepam, Amobarbital and Phenobarbital as ligand and GABAA protein as receptor. Interaction energy analyses by molecular dockings studied through both packages are in good agreement and indicate that the receptor of GABAA interacts with the Linalool, linalyl acetate, lavandulyl acetate, $\alpha$-terpineol and geranyl acetate effectively and among which Diazepam and Geranyl acetate have very close and similar interactions with GABAA receptor. This behavior implies that the latest compound can do the same action as Diazepam Amobarbital and Phenobarbital does to alleviate and relief pain and act like a natural painkiller in body. The Lavandula angustifolia Mill compounds ligands are safer and milder with fewer or no side effects than the drugs currently used in the treatment of lessening high lessen Stress. Therefore angustifolia Mill compounds ligand can be better used for the development of new therapeutics to decrease formation of GABAA.

\section{ACKNOWLEDGMENTS}

This study was supported by the Young Research Club of Islamic Azad University, Shahrekord we thank him.

\section{REFERENCES}

1. Taylor RD, Jewsbury PJ, et al. A review of protein-small molecule docking methods, J. Compute.-Aided Mol. Des. 2002; 16:151-166.

2. Renaud ENC, Charles DJ J Essent. Oil Res. 2001; 13: 269.

3. Cavanagh HMA. Aust. Infect. Control. 2005; 10: 35 .
4. Moon T, Chan YF, et al. AICA National Conference. 2004; 46d.

5. Kenner D, Requena Y. Botanical medicine: a European professional perspective. Brookline, MA: Paradigm.

6. Kurioka S, Toshiaki K, et al. J Neurochem. 1981; 37: 421. 
7. Sieghart W. Adv Pharmacol. 2006; 54: 231.

8. David W, Rithcie, Evaluation of Protein Docking Predictions using Hex 3.1 in CAPRI rounds 1-2, Proteins, Structure, Fucntion and Genetics, Wiley-liss Inc.

9. Sternbach LH, Reeder E, et al. Metlesics, Quinazolines and 1, 4-benzodiazepines III substituted 2-amino-5-phenyl-3H-1,4benzodiazepine 4-oxides, J. Org. Chem. 1961; 26(11): 4488-4497.

10. Riss J, Cloyd J, et al. Collins, Benzodiazepines in epilepsy: pharmacology and pharmacokinetics. 2008; 118: 69-86.

11. Ritchie DW, Kemp GJL. Struct. Funct. Genet. 2000; 39: 178-194.
12. Morris GM, Huey R, et al. Olson, Autodock4 and AutoDockTools4: automated docking with selective receptor flexibility. J. Comput. Chem. 2009; 30: 2785-2791.

13. Cosconati S, Forli S, et al. Virtual screening with AutoDock: theory and practice. Expert Opin. Drug Discovery. 2010; 5: 597-607.

14. Forli S, Olson AJ. A force field with discrete displacement waters and desolvations entropy for hydrate ligand docking. Med. Chem. 2012; 55: 623-638.

15. http://www. rcsb. org/pdb/

16. http://www.arguaslab.com

17. Shukla MK, Leszcynski J, Chem. Phys. Lett. 2006; 48: 317. 